\title{
GROUND-STATE ENERGY OF A FRUSTRATED $\pm J$ ISING MODEL ON A STRIP OF SQUARES
}

\author{
M. BEASZYK AND B. Fechner \\ Institute of Physics, A. Mickiewicz University \\ Umultowska 85, 61-614 Poznań, Poland
}

\begin{abstract}
We investigated $a \pm J$ Ising model on an infinite strip of squares of the width of one and two bonds with a random distribution of ferro- and antiferromagnetic exchange interactions. The exact ground-state energy as a function of the concentration of antiferromagnetic bonds was derived and its singular points in the complex-plane were found.
\end{abstract}

PACS numbers: 05.50.+q, 75.10.Nr, 75.50.Lk

\section{Introduction}

An important approach in investigation of the phase transitions consists in studying models which are exactly solvable although not necessarily in the closed form. In this respect random spin systems offer more difficulties than their ordered counterparts for which the translational and point symmetries considerably reduce the mathematical calculations. However, it has been shown that some exact ground-state properties can be found for infinite strips of finite width with a random distribution of ferromagnetic $(+)$ and antiferromagnetic $(-)$ bonds [1-4]. The essence of the method of calculations used lies in finding all possible configurations of $(+)$ and $(-)$ bonds and evaluating their contribution to the energy. However, there is no way to give a simple algorithm to find them out as the width increases. In this paper we describe a calculation procedure in which the problem can be formulated in a matrix form and solved using algebraic methods.

\section{The model and the method of calculations}

At the beginning we consider a simple strip consisting of squares with a single bond, and assume that the energy is determined by the Ising Hamiltonian with a random distribution of ferromagnetic and antiferromagnetic interactions with the probabilities $1-p$ and $p$, respectively. Let us define an $n$-cluster as a sequence of $n$ squares of the same kind. For a strip of the width of one bond there exist two kinds of clusters: frustrated and unfrustrated ones. The frustrated cluster or the smallest sequence of clusters, whose contribution to the energy is additive, we shall call a block. 
Let $P_{\mathrm{u}}(z)$ be a generating function for unfrustrated squares. For $z=1$ it is equal to the probability of finding any sequence of unfrustrated squares. It can be written in a matrix form

$$
P_{\mathrm{u}}(z)=[p,(1-p)] \boldsymbol{F}\left(\sum_{n=1}^{\infty}(z \boldsymbol{U})^{n}\right) \boldsymbol{F}\left[\begin{array}{l}
1 \\
1
\end{array}\right],
$$

where $\boldsymbol{U}$ and $\boldsymbol{F}$ are the transition matrices for an unfrustrated and frustrated squares, respectively. If we assume that the left hand bound of a square in the cluster belongs to its neighboring square, then

$$
\begin{aligned}
& \boldsymbol{U}=\left[\begin{array}{cc}
(1-p)^{3}+p^{2}(1-p) & 2 p^{2}(1-p) \\
2 p(1-p)^{2} & p^{3}+p(1-p)^{2}
\end{array}\right], \\
& \boldsymbol{F}=\left[\begin{array}{cc}
2 p(1-p)^{2} & p^{3}+p(1-p)^{2} \\
p^{2}(1-p)+(1-p)^{3} & 2 p^{2}(1-p)
\end{array}\right] .
\end{aligned}
$$

If $P_{\mathrm{u}}(n)$ is the probability of finding an unfrustrated $n$-cluster then the contribution to the energy per spin resulting from unfrustrated squares is given by

$$
-\frac{3 J}{2} \sum_{n=1}^{\infty} n P_{\mathrm{u}}(n)=-\left.\frac{3}{2} J \frac{\mathrm{d}}{\mathrm{d} z} \sum_{n=1}^{\infty} z^{n} P_{\mathrm{u}}(n)\right|_{z=1}=-\left.\frac{3}{2} J \frac{\mathrm{d}}{\mathrm{d} z} P_{\mathrm{u}}(z)\right|_{z=1} .
$$

In the case of frustrated clusters, one has to distinguish even and odd clusters. For even clusters the contribution to the energy is equal to

$$
-\frac{2 J}{2} \sum_{n=2,4,6, \ldots}^{\infty} n P_{\mathrm{f}}(n)=-\left.J \frac{\mathrm{d}}{\mathrm{d} z} \sum_{n=2,4,6, \ldots}^{\infty} z^{n} P_{\mathrm{f}}(n)\right|_{z=1},
$$

while for odd clusters it is

$$
\begin{aligned}
& -\frac{J}{2} \sum_{n=1,3,5, \ldots}^{\infty}(2 n-1) P_{\mathrm{f}}(n)= \\
& -\frac{J}{2}\left[\left.2 \frac{\mathrm{d}}{\mathrm{d} z} \sum_{n=1,3,5, \ldots}^{\infty} z^{n} P_{\mathrm{f}}(n)\right|_{z=1}-\sum_{n=1,3,5, \ldots}^{\infty} P_{\mathrm{f}}(n)\right]= \\
& -\left.J \frac{\mathrm{d}}{\mathrm{d} z} \sum_{n=1,3,5, \ldots}^{\infty} z^{n} P_{\mathrm{f}}(n)\right|_{z=1}+\frac{J}{2} \sum_{n=1,3,5, \ldots}^{\infty} z^{n} P_{\mathrm{f}}(n), .
\end{aligned}
$$

where $P_{\mathrm{f}}(n)$ is the probability of finding a frustrated $n$-cluster. Taking into account both contributions one gets

$$
\frac{E}{J}=\frac{1}{2}\left(\frac{-14+7 x^{2}-5 x^{4}}{6-3 x^{2}+x^{4}}\right) \quad \text { with } \quad x=1-2 p .
$$

Now, we consider a strip of the width equal to a double square. In this case there exist four kinds of clusters. We shall denote by $n n$ a cluster with an upper and a lower squares unfrustrated, by $n f$ a cluster in which the upper square is unfrustrated and the lower one frustrated and so on for $f n$ and $f f$. If we assume that the clusters do not interact with each other, then they contribute to the 
energy in the same way as the blocks, so the calculations of the energy can follow along the lines shown for a single strip.

In order to take into account the interactions, one has to notice that a single odd $f n$ or $n f$ cluster has one frustrated square with an unfrustrated bond at the edge of the strip. These clusters can form pairs. This happens in the case when between them there is a specific sequence of clusters which we shall call a conductive cluster. It consists of even $n f, f n$, and $f f$ clusters. Now, let us define a conductive block as any sequence of odd $n f$ or $f n$ clusters separated by a conductive cluster. For such a block, we introduce a generating function $G(z)$ that for $z=1$ is equal to the probability of finding a conductive block in the system. The exponent of $z$ is equal to the number of conductive clusters in the block increased by 1 . Thus the derivative $\left.\frac{\mathrm{d} G}{\mathrm{~d} z}\right|_{z=1}$ is equal to the mean number of odd $n f$ and $f n$ clusters. The generating functions for even and odd numbers of clusters are

$$
G^{\mathrm{e}}(z)=\frac{1}{2}[G(z)+G(-z)], \quad G^{\mathrm{o}}(z)=\frac{1}{2}[G(z)-G(-z)],
$$

respectively. Odd clusters of the type $n f$ and $f n$ can be arranged in pairs. The mean number of such pairs per a pair of squares (i.e. for each three sites) for even and odd number of clusters can be found from $\left.\frac{1}{2} \frac{\mathrm{d} G^{\circ}(z)}{\mathrm{d} z}\right|_{z=1}$ and $\left.\frac{1}{2} \frac{\mathrm{d}}{\mathrm{d} z}\left(\frac{1}{z} G^{\circ}(z)\right)\right|_{z=1}$, respectively. So, in order to calculate the contribution to the energy per site, one has to multiply the mean number of pairs by $-\frac{1}{3} J$. In this case we can calculate it from

$$
-\left.\frac{1}{3} J\left\{\frac{\mathrm{d} G^{\mathrm{e}}(z)}{\mathrm{d} z}+\frac{\mathrm{d}}{\mathrm{d} z}\left[\frac{1}{z} G^{\circ}(z)\right]\right\}\right|_{z=1} .
$$

Finally we obtain the energy in the following form:

$$
\frac{E}{J}=\frac{-120-16 x^{2}-145 x^{4}+52 x^{6}-89 x^{8}+8 x^{10}-7 x^{12}-4 x^{14}+x^{16}}{12\left(8+7 x^{4}-2 x^{6}+3 x^{8}\right)} .
$$

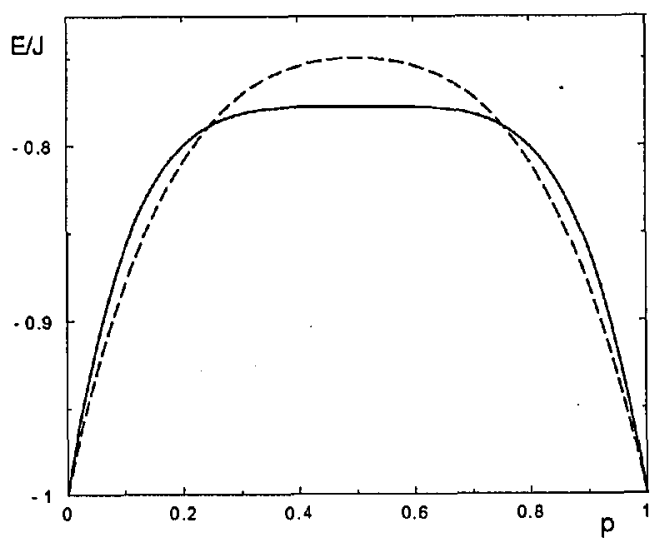

Fig. 1. The energy per bond vs. concentration of antiferromagnetic bonds $p$ : dashed line - singel strip, full line - double strip. 
The above calculation procedure can be applied to a strip of any width. In the case of three squares, the number of different kinds of clusters and rank of the transition matrices are equal to 8 and one has to deal with matrices with 64 rows and columns.

The ground-state energy for the strip of the width equal to one and two squares is shown in Fig. 1. Its singular points, which as the width increases to infinity can indicate a phase change, have been found. For the upper, right hand side of the complex plane they are: for the strip of one bond width $z=1.405+$ $0.689 \mathrm{i}$, for the strip of two bonds width $z_{1}=0.5959+0.8587 \mathrm{i}, z_{2}=1.0513+0.6241 \mathrm{i}$.

\section{References}

[1] B. Derida, J. Vannimenus, Y. Pomeau, J. Phys. C 11, 4749 (1978).

[2] T. Morita, T. Horiguchi, J. Phys. C 13, 6483 (1980).

[3] B. Fechner, M. Błaszyk, J. Phys. C 19, 2785 (1986).

[4] T. Kadawaki, Y. Nonomura, H. Nishimori, J. Phys. Soc. Jpn. 65, 1609 (1996). 